\title{
La evaluación de diseños de intervención para el Prácticum como instrumento de formación e investigación en el Máster de Profesorado de Ciencias Sociales.
}

\section{Assessment of intervention designs in the Practicum as training and research instru- ment in the Master's Degree in Teachers Trai- ning of Social Sciences}

\section{Dr. Daniel David Martínez Romera} Universida de de Málaga, España.

Martínez Romera, D.D. (2017). La evaluación de diseños de intervención para el Prácticum como instrumento de formación e investigación en el Máster de Profesorado de Ciencias Sociales.Revista Prácticum, Vol 2(2) 32-49. ISSN 2530-4550 


\section{Resumen}

La presente investigación intenta comprobar la pertinencia de combinar el método de evaluación de $360^{\circ}$ con la rúbrica digital federada en los procesos de evaluación del profesorado en formación, con objeto de encontrar formas eficientes de identificar patrones de comportamiento y variables significativas que ayuden a la comprensión de las dinámicas internas en pequeño y gran grupo; así como dotar al alumnado, desde la experiencia en los seminarios previos al prácticum, de elementos de juicio fundados respecto a su futura labor docente. La investigación fue llevada a cabo sobre un grupo de 26 profesores en formación, responsable tanto de construir la rúbrica de evaluación para la exposición de los trabajos finales en pequeño grupo como de aplicarla. El desarrollo práctico de la metodología permitió detectar algunas relaciones singulares individuo/grupo/clase, así como facilitó a los futuros docentes vivir en primera persona estrategias de evaluación pertinentes para la fase de prácticum, dotándose de este modo tanto de una metodología como de una valoración fundada al respecto. Aunque en un estadio todavía en desarrollo, la integración de métodos parece abrir nuevas fórmulas de seguimiento y evaluación docente; por ello, consideramos que seguir investigado en esta línea permitirá definir estrategias más completas de trabajo y evaluación en contextos de formación docente.

\section{Abstract}

The present research tries to verify the pertinence of combining $360^{\circ}$ evaluation method with federated digital rubric in evaluation processes of teacher training, in order to find efficient ways of identifying behavior patterns and significant variables that help the understanding of internal dynamics in small and large group; as well as providing the students, from the theoretical classroom experience prior to the prácticum, with well-founded elements of judgment regarding their future teaching work. The research was carried out on a group of 26 teachers in training, responsible both to construct the rubric of evaluation for the exhibition of the final work in small group and to apply it. The practical development of the methodology allowed to detect some unique individual / group / class relationships, as well as it helped the future teachers living in first-person evaluation strategies for the practice phase, giving them both a methodology and a valuation founded about it. Although at a stage still in development, the integration of methods seems to open new formulas of monitoring and evaluation; therefore, we believe that further research in this line will allow defining more complete strategies of work and evaluation in contexts of teacher training.

\section{Palabras claves:}

Evaluación, prácticum, ciencias sociales, didáctica, innovación tecnológica, investigación educativa

\section{Key words:}

Evaluation, prácticum, social sciences, didactic, technological innovation, educational investigation 


\section{Introducción y estado de la cuestión}

La importancia de la formación del profesorado de secundaria en España queda bien ilustrada con su transformación, en tanto que educación reglada, desde un curso menor de posgrado complementario para cada titulación académica a un Máster Interdisciplinar de curso completo y entidad propia (Lorenzo-Vicente, Muñoz-Galiano, \& Beas-Miranda, 2015; Perales-Montolío, Jornet-Meliá, \& González-Such, 2014; Viñao, 2013; González-Faraco, Pérez-Moreno, \& Jiménez-Vicioso, 2011; Esteve-Zarazaga, 2009). Entre los factores educativos que sostienen dicha evolución destacan la búsqueda de la excelencia académica, la adquisición de mayor experiencia práctica en los centros y un mayor dominio de los recursos y habilidades docentes, especialmente los tecnológicos (Pérez-Pueyo, Hortigüela-Alcalá, \& Gutiérrez-García, 2016; SerranoRodríguez \& Pontes-Pedrajas, 2015; Valdés-Puentes, Bolivar-Botia, \& Moreno-Verdejo, 2015).

Se abre así una ventana de oportunidad para consolidar las didácticas específicas como puente entre ciencia y educación, lo que obliga a poner sobre la mesa tres cuestiones clave a nuestro entender: profundizar en la integración de conocimientos que mejoren el acto educativo, investigar y comprobar su utilidad práctica y desarrollar un corpus teórico básico que articule la coherencia de la disciplina.

La interacción e integración de conocimientos es algo común en la frontera entre ciencias, allá donde una única perspectiva o marco de referencia se muestran insuficientes para explicar los hechos u ofrecer soluciones viables. Sin embargo, cuando dicha mixtura implica una ciencia específica y la pedagogía, la psicología de la educación, o la educación como materia general, el recelo apriorístico de la innovación o la investigación se convierte en la primera dificultad a superar. Por tanto, demostrar la necesidad de la investigación epistemológica y su solvencia intelectual mediante la poiésis (Morin, 2001; Morin, 1999) de teorías, métodos y técnicas se postula como el mejor camino para reivindicar su pertinencia.

Este esfuerzo no debe producirse en un marco puramente teórico, ya que el objetivo fundamental que es mejorar el acto educativo es intrínsecamente práctico. Por ello, investigar y comprobar con datos la eficacia de teorías, métodos y técnicas se convierte en el otro gran brazo sobre el que construir el discurso didáctico, el de los resultados (Aguilar-Cuesta \& Martínez-Romera, 2017; Curiel-Marín \& Fernández-Cano, 2015).

La articulación de la formación teórica y práctica en propuestas que sean contrastables y participativas para los futuros docentes es un foco de interés de primer orden en todas las Facultades de Educación (Sepúlveda, M.P., Gallardo, M., Mayorga, M.J. y Madrid, D. 2017; Martínez, A. et al., 2016; Salerni, 2016; Alsina, Á. y Batllori, R., 2015). Es en este sentido que cobra especial relevancia la utilización de metodologías de forma transparente, esto es, haciendo partícipes a docente y discente del proceso, en este caso de evaluación. Algo que queda reflejado por la recurrencia de los estudios y trabajos de valoración y revisión sobre el grado de éxito en la construcción del puente teoría práctica tanto en los Grados de Primaria como en el Máster de Profesorado (FuentesMoreno, 2016; Cachón-Zagalaz, J. et al., 2015; González-Ballesteros, M. y FernándezLozano, P., 2014).

La consolidación del discurso didáctico y su fundamentación rigurosa ayuda a definir mejores estrategias y nuevas líneas en las que explorar, garantizando así que el fin último de su existencia es ayudar a hacer más comprensible y aprehensible el conocimiento científico específico, tanto por el beneficio educativo que supone en sí mismo como por el fortalecimiento de los instrumentos intelectuales de la futura ciudadanía. 
La evaluación de diseños de intervención para el Prácticum como instrumento de formación e investigación en el Máster de Profesorado de Ciencias Sociales.

La presente investigación centrará sus esfuerzos fundamentalmente en la segunda clave, en el contexto de los procesos de evaluación. Las aportaciones actuales en este campo son cada vez más profusas (Arribas-Estebaranz, Manrique-Arribas, \& Tabernero-Sánchez, 2016; Cabero \& Barroso, 2016), si bien el tratamiento específico de las rúbricas (Martínez-Romera, Cebrián-Robles, \& Cebrián-de-la-Serna, 2016; PérezTorregosa et al., 2016; Cano, 2015; Cebrián-de-la-Serna, Cebrián-Robles, \& SerranoPuerto, 2015; Cebrián-de-la-Serna, Manuel \& Bergman, 2014; Cebrián-de-la-Serna, Manuel, 2014) es claramente más dinámico, en términos académicos, que el de la evaluación de $360^{\circ}$ (Bisquerra-Alzina, Martínez-Olmo, Obiols-Soler, \& Pérez-Escoda, 2006; Brutus \& Gorriti, 2005), algo que se explica en parte por el mayor consumo de tiempo que implica ésta en su aplicación y análisis.

La rúbrica ha demostrado ser un instrumento muy adaptable a diferentes estrategias y métodos (colaborativo, cooperativo, caso...), como lo refleja la multiplicidad de estudios y propuestas que la abordan desde la perspectiva del prácticum. Así, en el último Symposium Internacional sobre el Prácticum y las Prácticas Externas (http:// reppe.org/poio/) encontramos referencias al uso práctico de la rúbrica que podemos glosar en cuatro categorías:

- Instrumento clave en el desarrollo de un portafolio multimedia de calidad (Cebrián de la Serna, M.).

- Instrumento de autoevaluación discente y evaluación docente en la redacción de informes de prácticum (Arias Gago, A. R. et al.).

- Instrumento para la evaluación de contenidos y experiencias prácticas (Coiduras, J. L. et al.).

- Instrumento para la evaluación de competencias personales y prosociales durante el prácticum (Peire Fernández, T. et al.; Pérez-Torregosa, A. B. et al.).

Por supuesto, todo ello, sin obviar la relevancia manifestada al respecto en otros eventos nacionales e internacionales como: III International Congress Corubric16, VI Seminario Internacional: Tecnologías para la evaluación de los aprendizajes o 1st International Conference on Higher Education Advances. Lo que nos permite afirmar que se trata de un instrumento relevante en el seno de la comunidad educativa especialmente adecuado a contextos prácticos.

En el caso que nos ocupa, tal vez su virtud más destacable sea la de facilitar la reintroducción en el acto educativo, durante su construcción en el aula, de elementos propios del discurso científico, que pudieran quedar silenciados de otro modo, tales como: la claridad, en oposición a la ambigüedad semántica que con frecuencia surgen en los juicios de valor; la pertinencia, que permite construir proposiciones de referencia acordes a la intencionalidad establecida; y la factibilidad, en tanto que ayuda a reflexionar si es posible, o razonable, intentar la medición de un aspecto en un contexto dado (Cohen, Manion \& Morrison, 2011).

La evaluación de $360^{\circ}$, por su parte, ha sido ampliamente utilizada en contextos profesionales, subrayando con ello su adaptabilidad, y sobre todo la importancia de su razón de ser: la evaluación integral de las competencias presentes en un grupo dado, enfrentando el autoconcepto con el de los pares y del propio docente. Con ello se posibilita el desarrollo del conocimiento propio en contextos de trabajo cooperativo o colaborativo, habituales hoy día tanto en el ámbito educativo como social (TejadaFernández, 2011). 


\section{Material y métodos}

La investigación se desarrolló en un grupo de clase formado por 26 personas, organizadas en 7 grupos no dirigidos de trabajo, integrados por 3 o 4 miembros, procedentes de diversas especialidades (Geografía, Historia, Historia del Arte y Filosofía), en la asignatura de Iniciación a la Investigación e Innovación Docente de la especialidad de Ciencias Sociales del Máster de Profesorado de la Universidad de Málaga, durante el curso 2016/17.

El objetivo didáctico de la tarea, que define el contexto de aprendizaje, fue acercar al alumnado la complejidad del proceso evaluador y sus dificultades, especialmente la necesidad de establecer criterios imparciales, observables y comprobables de lo que se pretende medir. Mientras que el objetivo de investigación se centró en verificar la capacidad de los instrumentos utilizados para identificar patrones o comportamientos internos en cuanto al desempeño individual y colectivo del alumnado.

El contexto curricular de la propuesta planteó el uso del trabajo final, en pequeños grupos no dirigidos, como experiencia previa de los discentes en el diseño y aplicación de metodologías didácticas pertinentes para la enseñanza de las Ciencias Sociales. Se asumía una estrategia diferente en cada grupo (Aprendizaje-Servicio, InvestigaciónAcción, Clase Invertida, ABP...), sobre la que debían elaborar tanto un diseño formal de intervención documental como llevar a cabo una intervención orientada a la ejecución práctica de la misma a partir de la simulación en clase.

La estructura de los documentos era condicionada por la naturaleza del método elegido, mientras que las exposiciones debían respetar una estructura general común: introducción y contextualización teórica sobre el método y el tema elegidos; ejecución en clase de una parte de la propuesta; conclusiones y análisis DAFO de la experiencia por parte del grupo expositor; pequeño debate, basado en la misma metodología, con el resto de clase respecto a la pertinencia de la didáctica usada; y, finalmente, la evaluación siguiendo la presente metodología.

La recogida de datos se planteó en entorno TIC mediante el uso de rúbricas federadas online, en tanto que garantizan el acceso en todo momento y lugar; fue seleccionada para ello una aplicación gratuita, CoRubric (Corubric.com), que permite además la recopilación de datos cualitativos (mediante anotaciones en el diseño) además de los cuantitativos (mediante su aplicación).

Para el análisis cuantitativo se recurrió a hoja de cálculo, en la que se desarrollaron procedimientos generales de agregación/segregación de variables mediante tablas dinámicas y la obtención de estadísticos elementales como frecuencias, medias aritméticas y desviaciones estándar; se hizo lo propio con Atlas.ti para el análisis semántico de las anotaciones así como para la detección de palabras clave de frecuencia marginal (Schmitt \& Schmitt, 2014); y a Weka para realizar la discretización de variables y la minería de datos de las evaluaciones, fundamentalmente para la exploración de reglas apriorísticas de asociación para la determinación de patrones ocultos (Agrawal \& Srikant, 1994; Liu, Hsu, \& Ma, 1998). 
La evaluación de diseños de intervención para el Prácticum como instrumento de formación e investigación en el Máster de Profesorado de Ciencias Sociales.

Para el desarrollo de la experiencia fue necesario el uso de aula TIC en dos sesiones monográficas de trabajo con CoRubric, elegida por tratarse una aplicación gratuita que permitía diferentes funciones que soportaban el modelo pedagógico diseñado. El resto del proceso colaborativo de grupo/clase se llevó a cabo en aula normal, bien como preludio de la primera sesión TIC o sincretización tras la segunda. Con objeto de garantizar una correcta sistematización de la investigación (Wood \& Smith, 2016; Egbert \& Sanden, 2014), se planteó una secuencia de trabajo en cinco fases:

Sesión previa al primer día de trabajo TIC, a tres semanas del inicio de exposiciones. Se plantea a la clase el objeto y alcance de la tarea a desarrollar, esto es, la construcción colaborativa del instrumento para evaluar las exposiciones grupales, contempla cuatro niveles: autoevaluación, evaluación entre los miembros del grupo, evaluación del resto de la clase y evaluación docente. Cada grupo será evaluado, así, de forma colectiva e individual. En todos los casos, se garantiza el anonimato de los evaluadores, de modo que la persona evaluada solo tiene acceso a una calificación media en función del número de evaluaciones recibidas. Se ejemplifica visualmente el instrumento con propuestas no relacionadas en su objeto, para evitar condicionamiento inicial.

Primera sesión de trabajo TIC con CoRubric, a tres semanas del inicio de exposiciones. Cada persona utiliza su ordenador de trabajo, durante la primera parte de la sesión, para construir una propuesta de rúbrica de evaluación mientras aprende el uso de la aplicación. Los elementos que debe contemplar son claramente explicitados: categorías, variables y evidencias semánticas asociadas a escala de medición. La segunda parte de la clase se vuelve a subdividir en dos: un primer momento para invitar y ser invitado a las rúbricas, con objeto de visitar otras propuestas y poder realizar comentarios y anotaciones de carácter cualitativo que ayuden a mejorarlas; y finalmente, realizar una puesta en común sobre los elementos elegidos y su frecuencia (a modo de histograma). Entre ésta y la siguiente sesión se deja un tiempo para la reflexión y las anotaciones entre compañeros, como fase no supervisada de dinámica interna.

Segunda sesión de trabajo TIC con CoRubric, a dos semanas del inicio de exposiciones. El alumnado vuelve a sentarse frente a su propuesta con dos indicaciones clave: tener en cuenta la síntesis realizada al final de la sesión anterior con objeto de que inicie un proceso de prelación y normalización propio de categorías, variables y evidencias; y añadir una ponderación relativa tanto para categorías como variables. En la segunda parte de la exposición, más extensa en esta ocasión, se vuelve a realizar una puesta en común, primero en pequeño grupo y luego en general, que debe llevar, necesariamente, a un consenso: la rúbrica colaborativa unificada con categorías y variables ponderadas, así como las evidencia definidas semánticamente de forma unívoca, factible y pertinente (Quivy \& Van-Campenhoudt, 2005) para todos los valores de la escala. El docente se hace responsable de registrar el resultado del proceso y elaborar la correspondiente rúbrica.

Sesión final, a una semana del inicio de exposiciones. Se realiza la exposición pública de la rúbrica unificada construida por el docente según los datos recogidos de la sesión anterior y se somete a escrutinio final por parte de la clase. En caso de haber matices o pequeñas mejoras, éstas han de quedar perfectamente indicadas para que se incluyan antes de su aplicación. El docente adquiere el compromiso de hacerla pública, y el alumnado el de inscribirse en ella, con anterioridad al inicio de las exposiciones. 
Fase de aplicación, durante el periodo de exposiciones. En caso de no disponer de un dispositivo electrónico (tablet, móvil, portátil...) el alumnado tomará, al igual que el docente, las notas que consideren oportunas para poder rellenar con posterioridad las diversas evaluaciones del día, ya sea sobre sí mismos y sus compañeros, si les ha tocado exponer, o sobre las exposiciones (evaluando tanto al grupo en conjunto, con un objeto de evaluación creado al efecto, como a cada uno de sus miembros). Como expresión final del proceso, esta fase hace posible la obtención de información sobre autopercepción, percepción de grupo de trabajo, percepción entre iguales (clase) y docente. Esto supone añadir un punto de vista adicional, el segundo, respecto a la concepción clásica de la evaluación de $360^{\circ}$, pero se considera interesante explorar dicho punto de vista en el contexto de la investigación. El instrumento aplicado queda ilustrado en la Ilustración 1.
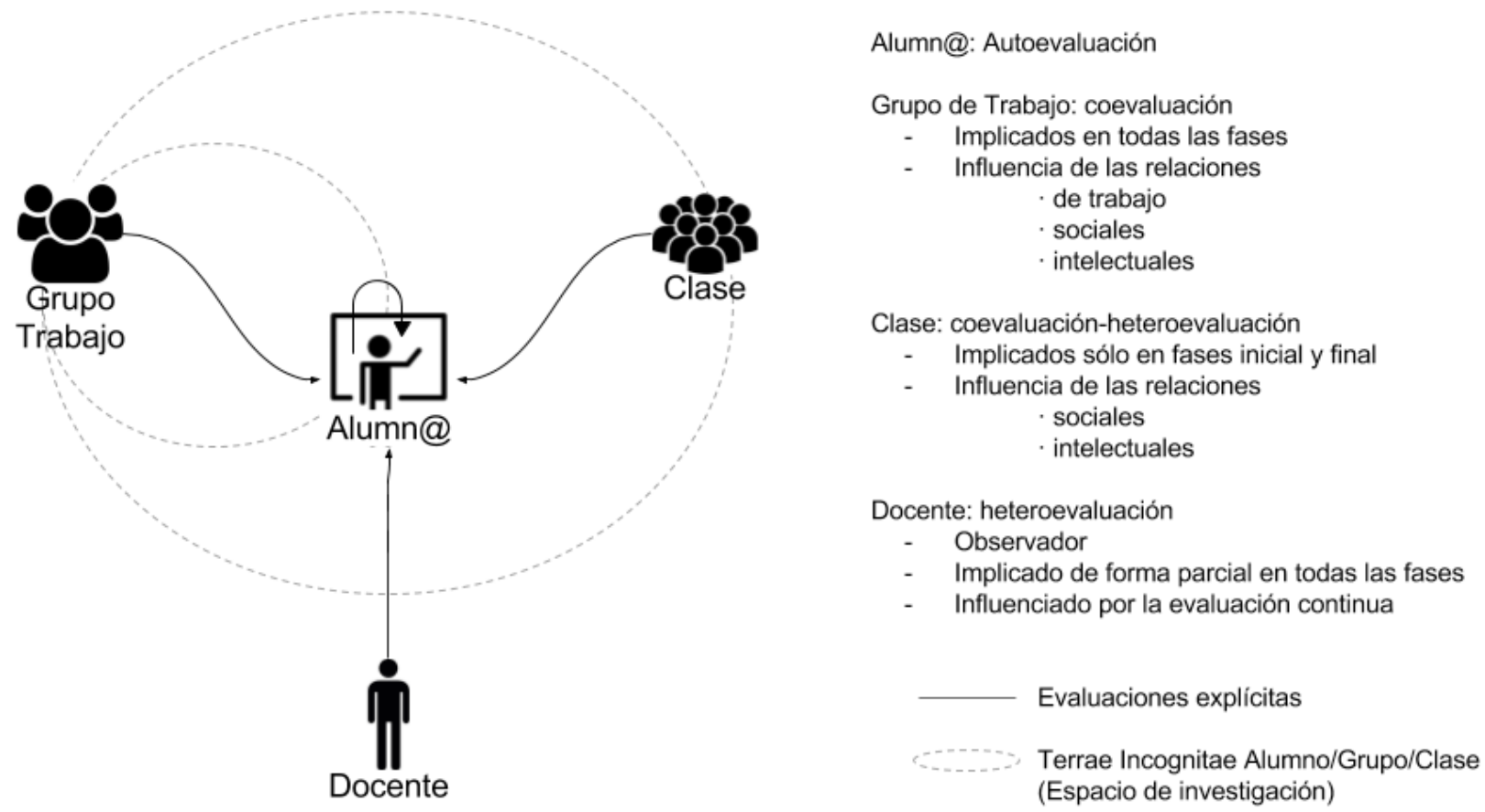

Gráfico 1. Variante de la Evaluación de $360^{\circ}$ aplicada en la práctica. Fuente: autor.

Dado que uno de los objetos de la propuesta es comprobar la eficacia de la combinación de los instrumentos educativos propuestos para determinar con mayor precisión el trabajo individual en contextos grupales, centraremos el análisis en los trabajos realizados con las anotaciones y las calificaciones emitidas. La hipótesis de trabajo será la siguiente: la utilización combinada de evaluación de $360^{\circ}$ y rúbrica digital ayudará a detectar dinámicas internas individuo/grupo/clase que de otro modo pasarían desapercibidas, lo que redundará en una mayor capacidad explicativa de las evaluación entre actores. 
Por otro lado, la propia medición realizada para contrastar la hipótesis constituye un elemento de reflexión sobre el grado de compromiso discente con la tarea, en términos de amplitud de la dispersión respecto a la media sobre los diversos aspectos evaluados. Lo que es, como mínimo, un indicio del grado de madurez con el que los futuros docentes de ciencias sociales se enfrentan a una de las tareas para las que están menos preparados en la práctica, evaluar; así como constituye una primera experiencia puente entre los fundamentos teóricos recibidos al respecto durante la fase de formación teórica y la necesidad de aprender a aplicarlos durante su desempeño en el prácticum. Se construye de este modo un laboratorio de experiencias dentro del aula que pretende fortalecer habilidades necesarias en la siguiente fase, y hacer posible la construcción del criterio propio respecto a la Didáctica de las Ciencias Sociales por parte de los docentes en formación.

\section{Análisis y resultados}

De los 26 miembros de clase, 16 (61.5\%) utilizaron finalmente la herramienta de anotaciones de CoRubric, generando un total de 50 anotaciones; su tipología, según categoría de destino y frecuencia, afecta a 12 de los 15 elementos de la rúbrica, La versión final del instrumento puede consultarse en el Anexo I. El 56.25\% (34.6\% del total) realizaron al menos 2 comentarios, lo que constituye una primera estimación sobre el interés individual respecto al instrumento de evaluación de las exposiciones. Partiendo de los datos representados en la Gráfica 1, si se agrega el número de miembros de cada grupo participante, y ordena por frecuencia absoluta de anotaciones, se obtiene: $\mathrm{B}(3 / 3 ; \mathrm{ni}=19), \mathrm{A}(3 / 4 ; \mathrm{ni}=10), \mathrm{E}(3 / 4 ; \mathrm{ni}=8), \mathrm{C}(2 / 4 ; \mathrm{ni}=6), \mathrm{D}(2 / 3 ; \mathrm{ni}=3), \mathrm{F}(2 / 4$; $\mathrm{ni}=3), \mathrm{G}(1 / 4 ; \mathrm{ni}=1)$.

Así, el $38 \%$ es realizado por un único grupo, B, en el que además participan sus tres miembros, lo que constituye una evidencia clara de implicación individual y colectiva; permite observar comportamientos marginales más interesantes, como el caso del grupo A, segundo en frecuencia, pero en el que 1 de sus 3 participantes realiza el $80 \%$ de las anotaciones del grupo.

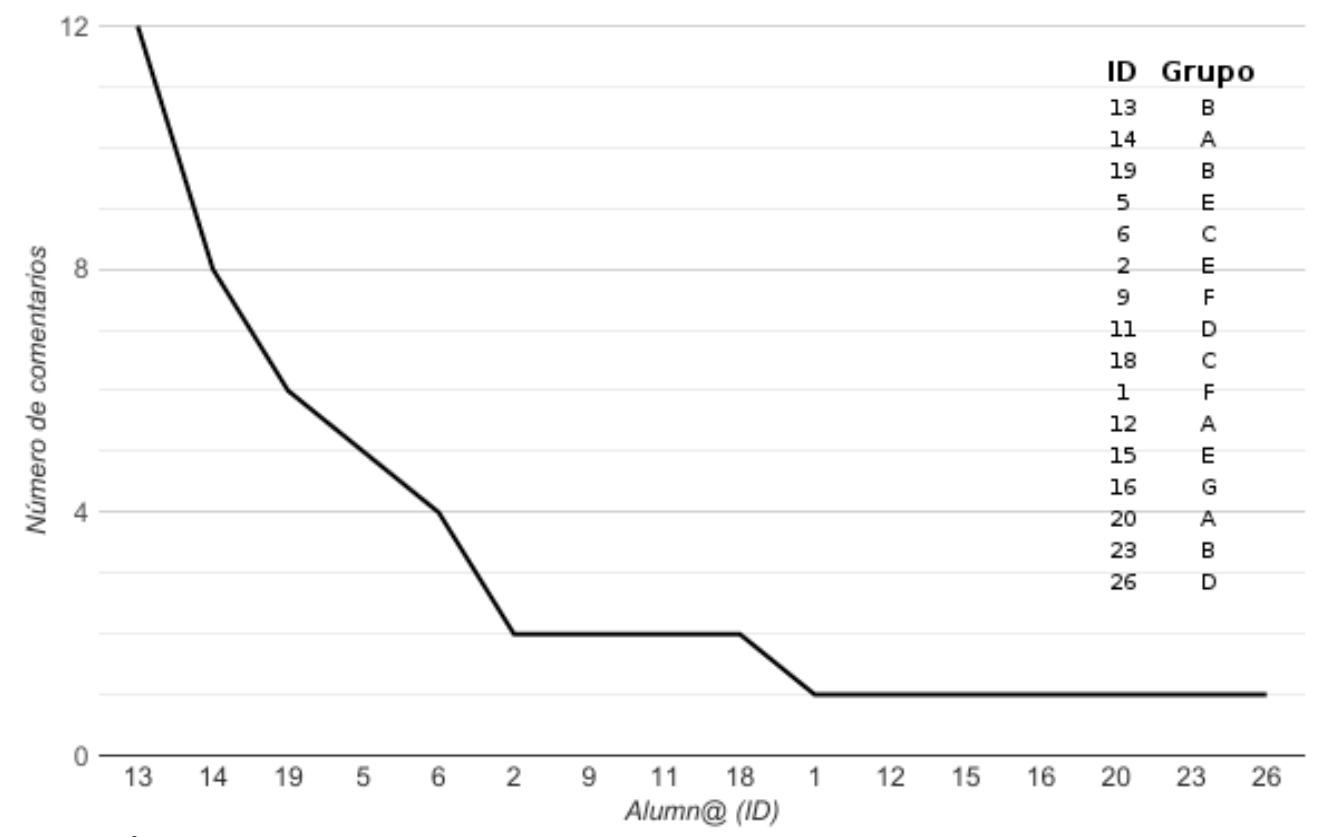

Gráfico 2. Frecuencia absoluta de comentarios por alumno. Fuente: autor. 
El análisis cualitativo arrojó una matriz de 565 palabras únicas, con frecuencias que oscilan entre 1 y 112 . Esto indica que pese a la escasa presencia de comentarios la redacción de los mismos es relativamente elaborada, con un promedio de 200 caracteres por anotación. La discriminación de significantes permitió detectar algunas palabras clave en el umbral de las frecuencias raras y muy raras (Laufer \& Ravenhorst-Kalovski, 2010):

- 1\% ( $\mathrm{f}<26)$ : metodología, seguridad, tiempo, alumnado, gestión, evaluación, creatividad, calificación, involucrar, dinamismo, conocimientos, adecuación.

- 0.1\% ( $\mathrm{f}<3)$ : valorar, utilización, innovación, adaptación, método.

Esta serie de conceptos demostró ser relevante tanto en la revisión personal de las propuestas como en el segundo debate grupal sobre la forma y contenidos del instrumento, evidenciándose una mayor influencia de estos perfiles en su refinamiento; de hecho, la principal área de discrepancia refleja una clara relación de identidad con buena parte de los conceptos incluidos en las series, lo que se ilustra con el siguiente ejemplo:

1.4 TIC y recursos utilizados ( $18 \%$ de todas las anotaciones). En esta categoría las frases utilizadas se apoyan en claves como creatividad, involucrar, utilización e innovación. Comentario de ID 13 realizado en la plataforma:

«Una buena utilización junto a la metodología son las variables más importantes para una buena utilización. Esta variable puede ponerse en práctica todos los conocimientos con respectos a las TICS que anteriormente no podía contener el alumnado. Por ejemplo, la innovación en el método como la utilización de TICs novedosas son variables que se han formado en el aula. Sin embargo, otras variables como expresión oral y corporal pueden que las pueda tener en la personalidad de la persona en sí misma».

Se han subrayado las coincidencias exactas, y resaltado con fondo gris las semejanzas semánticas de alta probabilidad (innovación y alumnado, respectivamente).

La matriz de datos recogida alberga 598 evaluaciones sobre una expectativa de 858 (26 personas x 33 personas+grupos), lo que implica una cobertura del 69.7\%. Usando como base el resumen estadístico de la matriz de datos, ilustrado en la Tabla 1 , el defecto del $30.3 \%$ es explicado por la desviación en el número de evaluaciones emitidas por persona, que a pesar de obtener una cobertura general elevada tiene dos extremos incuestionables: 8 de las 26 personas realizaron la tarea completamente (30.8\%) y 2 no la cumplimentaron en absoluto $(7.7 \%)$; quienes la realizaron totalmente pertenecen a los grupos $\mathrm{G}(3 / 4), \mathrm{C}(2 / 4), \mathrm{A}(2 / 4)$ y $\mathrm{E}(1 / 4)$, y quienes no la realizaron a los grupos $E(1 / 4)$ y $D(1 / 3)$. Si centramos la atención en el tipo de evaluación, se comprueba que 6 personas no se autoevaluaron, por grupos $\mathrm{F}(2 / 4), \mathrm{E}(2 / 4), \mathrm{C}(1 / 4)$ y $\mathrm{D}(1 / 3)$, y que un grupo, el $\mathrm{F}$, no evaluó a uno de sus miembros. 
La evaluación de diseños de intervención para el Prácticum como instrumento de formación e investigación en el Máster de Profesorado de Ciencias Sociales.

\begin{tabular}{|c|c|c|c|c|c|c|c|c|}
\hline \multirow[b]{2}{*}{ ID } & \multirow[b]{2}{*}{ Grupo } & \multicolumn{7}{|c|}{ Evaluaciones } \\
\hline & & Emitidas & Recibidas & $\begin{array}{c}\text { Ratio } \\
E / R\end{array}$ & $\begin{array}{c}\text { Auto } \\
(\%)\end{array}$ & Grupo (\%) & Clase (\%) & Profesor \\
\hline 1 & $F$ & 28 & 15 & 1,87 & 96,6 & 100,00 & 94,36 & 87,5 \\
\hline 2 & $E$ & 28 & 16 & 1,75 & 90,1 & 100,00 & 89,36 & 91,9 \\
\hline 3 & G & 33 & 15 & 2,20 & 92,1 & 100,00 & 94,32 & 84,0 \\
\hline 4 & $F$ & 17 & 13 & 1,31 & - & 91,10 & 90,88 & 94,0 \\
\hline 5 & $E$ & 0 & 15 & 0,00 & - & 89,35 & 77,99 & 76,4 \\
\hline 6 & $\mathrm{C}$ & 33 & 21 & 1,57 & 96,6 & 94,92 & 90,21 & 75,9 \\
\hline 7 & $F$ & 7 & 13 & 0,54 & - & 93,60 & 92,15 & 74,0 \\
\hline 8 & $\mathrm{C}$ & 33 & 22 & 1,50 & 91,75 & 94,15 & 91,24 & 91,6 \\
\hline 9 & $\mathrm{~F}$ & 29 & 13 & 2,23 & 93,5 & - & 84,79 & 89,2 \\
\hline 10 & $E$ & 6 & 16 & 0,38 & - & 96,05 & 90,09 & 71,5 \\
\hline 11 & $\mathrm{D}$ & 28 & 22 & 1,27 & 100 & 90,20 & 93,41 & 81,7 \\
\hline 12 & $\mathrm{~A}$ & 23 & 21 & 1,10 & 96 & 84,55 & 86,29 & 86,8 \\
\hline 13 & $B$ & 23 & 22 & 1,05 & 92,75 & 91,18 & 85,32 & 72,3 \\
\hline 14 & $\mathrm{~A}$ & 33 & 23 & 1,43 & 100 & 96,70 & 95,57 & 76,4 \\
\hline 15 & $E$ & 33 & 16 & 2,06 & 100 & 95,50 & 93,93 & 78,1 \\
\hline 16 & $\mathrm{G}$ & 33 & 15 & 2,20 & 94,6 & 96,70 & 90,19 & 81,5 \\
\hline 17 & $\mathrm{G}$ & 26 & 15 & 1,73 & 94,6 & 99,17 & 91,74 & 75,2 \\
\hline 18 & $\mathrm{C}$ & 18 & 22 & 0,82 & 97,5 & 92,40 & 90,44 & 88,9 \\
\hline 19 & $B$ & 27 & 21 & 1,29 & 97 & 94,38 & 85,77 & 92,1 \\
\hline 20 & $\mathrm{~A}$ & 15 & 21 & 0,71 & 100 & 83,80 & 87,35 & 88,0 \\
\hline 21 & $\mathrm{G}$ & 33 & 14 & 2,36 & 100 & 94,12 & 87,80 & 75,4 \\
\hline 22 & $\mathrm{~A}$ & 33 & 22 & 1,50 & 100 & 90,73 & 95,37 & 90,7 \\
\hline 23 & $B$ & 23 & 21 & 1,10 & 97,5 & 81,48 & 83,76 & 87,4 \\
\hline 24 & $\bar{D}$ & 0 & 20 & 0,00 & - & 89,15 & 86,10 & 87,0 \\
\hline 25 & $\mathrm{C}$ & 28 & 20 & 1,40 & - & 83,42 & 83,65 & 79,8 \\
\hline 26 & $\mathrm{D}$ & 8 & 17 & 0,47 & 93,6 & 89,60 & 93,08 & 74,9 \\
\hline \multicolumn{5}{|c|}{ Valoraciones media por grupo } & \multicolumn{4}{|c|}{ (б) } \\
\hline & A & & 22 & & $(2,00)$ & $88,95(6,03)$ & $91,30(5,01)$ & $89,35(4,48)$ \\
\hline & $\mathrm{B}$ & & 22 & & $(2,61)$ & $90,60(6,72)$ & $84,96(1,05)$ & $86,58(1,27)$ \\
\hline & $\mathrm{C}$ & & 20 & & $(3,09)$ & $90,02(5,31)$ & $88,99(3,52)$ & $82,58(9,04)$ \\
\hline & $\mathrm{D}$ & & 19 & & $(4,53)$ & $89,44(0,53)$ & $90,84(4,13)$ & $80,08(7,47)$ \\
\hline & $E$ & & 16 & & $(7,00)$ & $90,49(4,40)$ & $88,00(6,87)$ & $79,48(5,91)$ \\
\hline & $\mathrm{F}$ & & 14 & & $(2,19)$ & $91,23(4,59)$ & $90,69(4,10)$ & $82,68(7,63)$ \\
\hline & G & & 14 & & $(3,33)$ & $90,74(2,65)$ & $91,07(2,74)$ & $81,58(6,03)$ \\
\hline
\end{tabular}

Gráfico 3. Resumen estadístico por individuos y grupos de trabajo. Fuente: autor.

Se van detectando, de este modo, patrones de comportamiento en las relaciones del binomio individuo-grupo: 3 de los 4 miembros de $\mathrm{G}$ realizaron completamente la tarea y no presentan incidencias serias en la aplicación del instrumento, en menor medida algo similar ocurre con el grupo $A ; 2$ de los 4 miembros del grupo $F$ no realizaron su autoevaluación, y un tercero del mismo no fue evaluado por sus compañeros, algo que ocurre de forma más atenuada en el grupo D. Una tercera tipología es la representada por el grupo $\mathrm{E}$, en el que 1 de sus 4 miembros realizó completamente la tarea mientras que otro no la realizó en absoluto, si se revisa el comportamiento del resto de miembros, se comprueba una polarización similar ( $84.85 \%$ y $18.18 \%)$; la gran diferencia de comportamiento interno detectado podría llevar a pensar en conflicto, sin embargo un miembro cuenta con una puntuación perfecta $(100 \%)$ en la evaluación de grupo, algo que no se puede explicar en términos de disensión pero sí de comensalismo. Obtenemos con ello un primer indicio de la naturaleza de las relaciones internas del grupo. 
Si se discretizan las variables cuantitativas en 10 intervalos regulares, con objeto de mantener un sistema de categorías comparable, se puede realizar una análisis apriorístico basado en reglas. Esto nos permite determinar correlaciones en función de diferentes tipologías métricas (confianza, sustento, balance y convicción). A efectos analíticos nos apoyaremos fundamentalmente en la primera, en tanto que determina la proporción de ejemplos cubiertos por la premisa que también cubre sus consecuencias, esto es, su concomitancia. Se seleccionan a continuación algunas de las reglas más interesantes, de las 108 encontradas y exploradas para un umbral de confianza igual o superior a 0.6, que cubren diferentes puntos de vista analíticos, con objeto de ilustrar la versatilidad de la propuesta realizada:

- (39) Recibidas='(-inf-14]' $4==>$ Grupo=F 3 <conf:(0.75)> lift:(4.88) lev:(0.09) [2] conv:(1.69). Esta regla indica que si la persona está en el intervalo 1, el más bajo, de evaluaciones recibidas por parte de la clase, entonces con un $75 \%$ de seguridad pertenece al grupo F. Ya que sólo uno de las cuatro personas en Recibidas no pertenece a Grupo (valores contiguos a las variables). Esto constituye un indicador del interés y proximidad del resto de la clase hacia estas personas.

- (44) Grupo=G 4 ==> Emitidas='(29.7-inf)' 3 <conf:(0.75)> lift:(2.44) lev:(0.07) [1] conv:(1.38). Si se pertenece al grupo $G$, con un $75 \%$ de probabilidad se estará en el intervalo más alto de evaluaciones emitidas. En este caso se observa una regla que ayuda a discernir la implicación individual con la tarea, y que adquiere carácter grupal debido al número de miembros que la secundan.

- (48) Grupo=A $4==>$ porAuto='(99.175-inf)' 3 <conf:(0.75)> lift:(3.25) lev:(0.08) [2] conv:(1.54). Si se pertenece al grupo A, con un $75 \%$ de probabilidad se habrá hecho una autoevaluación perteneciente al intervalo más alto posible. Lo interesante de esta regla es que ha permitido discernir un comportamiento grupal sobre un hecho individual como el autoconcepto.

- (62) porProfe $={ }^{\prime}(9.18$-inf) $4==>$ porClase $=$ '(93.812-inf)' 3 <conf:(0.75)> lift:(3.9) lev:(0.09) [2] conv:(1.62). Una regla sobre concomitancia de evaluaciones. Cuando el docente ha emitido una nota en el intervalo máximo sobre la exposición realizada por un alumnado, en el $75 \%$ de las ocasiones la nota correspondiente de la clase también se ha ubicado en su intervalo máximo. La exploración de este tipo de reglas permite verificar el grado de convergencia sobre el objeto de evaluación.

Una forma gráfica, sintética y aproximada, de comprobar estos resultados es estudiar las diferencias entre los cocientes de relación de autoevaluación/clase y grupo/ clase, tal y como se ilustra en el Gráfica 2: 


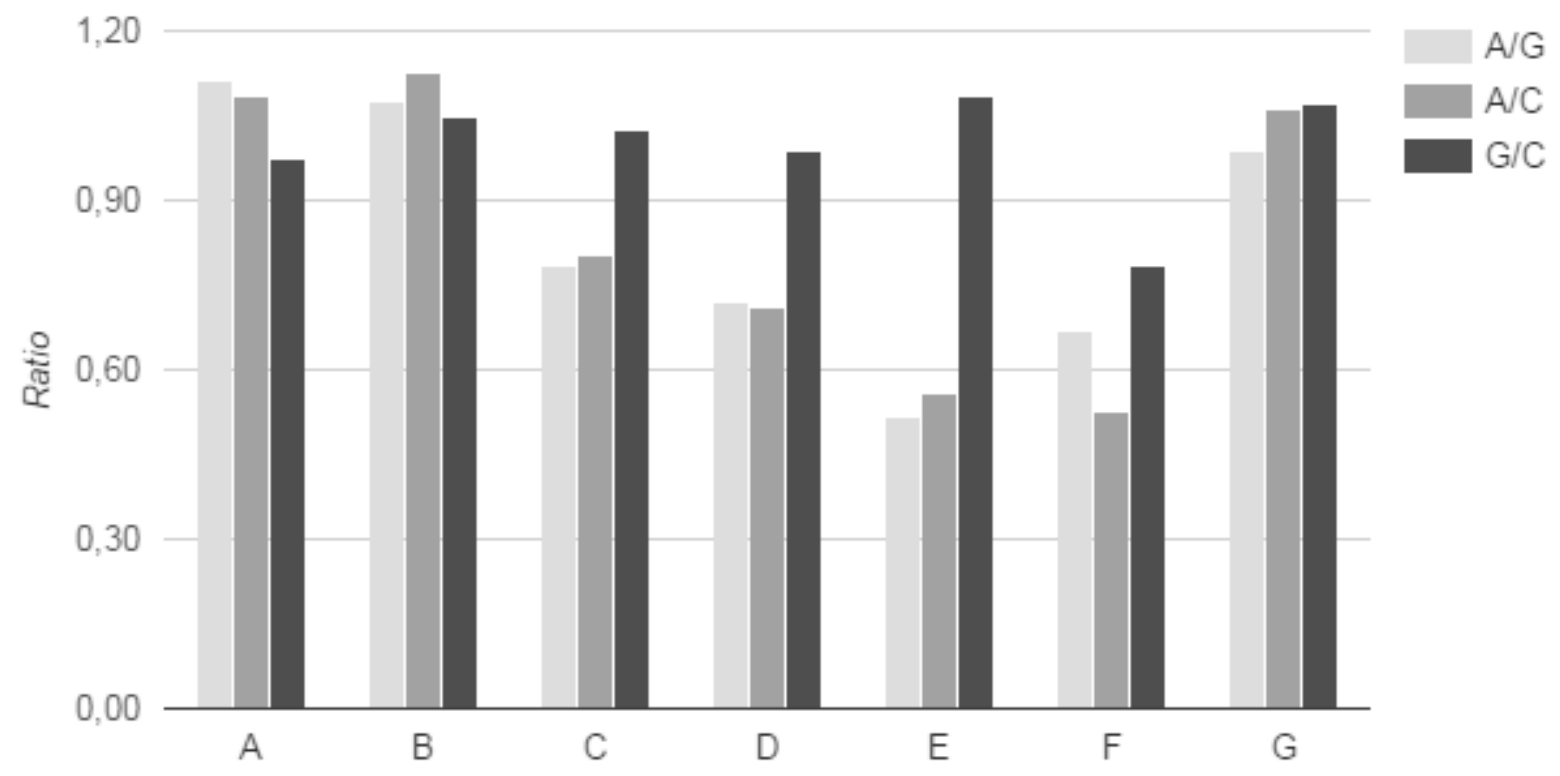

Grupos

Gráfico 4. Heterogeneidad en las calificaciones por ratios $(A) l u m n o /(G) r u p o,(A) l u m n o /(C) l a s e$ y (G)rupo/(C)lase. Fuente: autor.

Los grupos con mayor heterogeneidad entre sus indicadores (C, D, E y F) son los que al principio del análisis mostraron mayor número de incidencias, mientras que los restantes ( $A, B$ y $G$ ) exhibieron en términos generales valores elevados en cuanto a evaluaciones emitidas y recibidas, así como un menor número de incidencias por parte de sus miembros durante la fase de aplicación del instrumento. En conjunto, se detecta que los grupos cuyos miembros se implicaron más en el diseño de la rúbrica, e hicieron un uso más ajustado a lo esperado de la misma fueron, en general, también percibidos por la clase como más homogéneos, con la salvedad explícita del grupo A, en términos de desviación estándar de las calificaciones recibidas. Dicha homogeneidad no implica necesariamente un comportamiento de excelencia, si se desagrega por puntos de vista: el grupo B posee la nota mínima a criterio de la clase, siendo además el de menor desviación estándar; mientras que el criterio docente señala al grupo E como el valor menor en la exposición, y al C como el más heterogéneo. Se comprueban, en consecuencia, discrepancias respecto a los resultados sintéticos pero que en todo caso no llegan a contradecir el análisis de ratios.

Los grupos donde sólo parte de los miembros parece haber asumido el compromiso en todas las fases reflejan mayor número de incidencias y menor grado de cumplimiento de las tareas y reflejan una mayor dispersión de notas individuales respecto a la grupal; dicha falta de cohesión ha podido interpretarse en el caso del grupo E en términos de comensalismo antes que de enfrentamiento interno. También ha permitido detectar una situación de pequeño grupo que debe centrar el interés docente, ya que no pueden ser explicadas con los datos obtenidos, como sucede en $F$, donde uno de sus miembros no recibió calificación por parte de su equipo y al que la clase señaló con la nota más baja en la exposición del mismo. Finalmente, se puede realizar la identificación de las personas con mejor desempeño individual, que en el estudio lo ocupan ID5 como nota menor e ID14 como mayor, atendiendo al criterio de la clase; e ID18 e ID22 respectivamente para el criterio docente. 


\section{Discusión y conclusiones}

La construcción del pequeño laboratorio de prácticas dentro de la asignatura teórica como antesala del prácticum, que planteáramos en la metodología, nos parece útil como estrategia para encadenar e incardinar la concreción de la teoría y la explicación de la práctica. En este sentido, a pesar de las limitaciones que toda simulación presenta, la participación activa, la asunción de la propia responsabilidad en la emisión de un juicio que tendrá sus efectos sobre terceros y la demostración factual de que los procesos de evaluación pueden y deben ser formativos, constituyen un aprendizaje pertinente para la fase de prácticum. Muy especialmente si se tiene en cuenta la trayectoria del alumnado en la especialidad durante su formación universitaria, que habitualmente no ha conocido otra forma, ni dimensión de la evaluación, que la cuantificación de su desempeño a partir de evaluación docente de prueba escrita.

La construcción colaborativa de la rúbrica ha permitido desarrollar un proceso temporal de trabajo presencial y no presencial del que el docente puede obtener indicios de implicación mediante la cantidad y calidad de las anotaciones realizadas. El pensamiento crítico del alumnado, consciente de la relevancia para su evaluación de la tarea, fue el que más influenció los aspectos semánticos y formales de la misma. Desde una perspectiva docente es interesante tener elementos objetivos en los que apoyar su juicio a la hora de discernir dinámicas internas y su impacto en la formación individual y el ritmo de clase. La introducción de la evaluación de $360^{\circ}$ en la aplicación de la rúbrica ha supuesto un valor añadido en los siguientes aspectos:

- $\quad$ El cotejo de cómo se ve a sí misma la persona que expone, cómo la ven sus compañeros de trabajo, cómo lo ve el resto de la clase, en su papel de público/ alumnado, y el docente como seguidor del desempeño individual y colectivo desde el inicio, hace posible detectar relaciones interpersonales internas al grupo y a la clase, de difícil aprehensión docente cuanto mayor es el número de estudiantes. Las discrepancias en los mismos a la hora de calificar a sus miembros o las diferencias entre el autoconcepto y la nota recibida por la clase son aspectos claros, que en sí mismos no suponen una explicación pero sí ayudan a la detección de elementos sobre los que centrar el interés docente.

- Como hemos tenido la oportunidad ilustrar, la falta de cumplimiento o compromiso en las distintas tareas incluida la evaluación es, en igualmente, un indicador para el docente. Ya sea por la ausencia de anotaciones, la baja tasa de evaluaciones emitidas o recibidas, e incluso por no haberse autoevaluado, o quedar una persona sin evaluar de forma sistemática. Así, la omisión también se constituye en dato sobre el que reflexionar y desarrollar estrategias exploratorias para discernir la interpretación más ajustada a la realidad del mismo.

- $\quad$ Permite realizar una minería de datos más amplia, en tanto que se incrementa el número de variables significativas y en consecuencia el número posible de relaciones principales o marginales, según el interés. En el caso estudiado, la parametrización del análisis de asociación detectó 108 reglas, cuatro de las cuales han sido comentadas por su alto interés ilustrativo y por representar relaciones que posiblemente no habrían sido detectadas de otro modo. Identificar patrones de comportamiento entre individuos, individuos y grupos o entre éstos últimos, se hace posible mediante el cruce de datos entre evaluadores y evaluados. 
Sin embargo, los hechos identificados no siempre pueden ser interpretados adecuadamente por falta de contexto. En este sentido, poder documentar mejor las relaciones entre los miembros de la clase se convierte en algo necesario. Incluir en esta propuesta metodológica una técnica de cualificación interpersonal del alumnado como el sociograma, al menos al inicio y final del procedimiento, podría ayudar de forma sustantiva a saber en qué sentido interpretar las discrepancias en las calificaciones.

Tampoco se explica la baja correlación, en términos individuales, entre la implicación de ID13, ID14 e ID19 en la fase de anotaciones/diseño y su baja correlación en la evaluación emitida por clase y docente, más allá de la influencia que tuvieron en la creación y ajuste de la rúbrica. Sin duda, introducir elementos exploratorios sobre perfiles docentes como el cuestionario Sternberg-Wagner (Sternberg, 1999), así como haber realizado una experiencia expositiva previa, que garantizase el utillaje básico para las personas menos diestras en la comunicación oral, son aspectos sobre los que reflexionar tanto por el factor ruido que introducen en el estudio como por sus implicaciones formativas en los discentes. Como ventaja, dichos instrumentos pueden tener cabida y pertinencia como parte de la asignatura citada, obteniéndose así una suerte de sinergia en términos curriculares y de tiempo.

Entendemos que la hipótesis formulada al inicio es sólo confirmada en su premisa inicial, en la medida que incluso los grupos más heterogéneos analizados valoran por encima de su media a los miembros que se mostraron activos en las fases previas; pero no se observa una correlación clara en la asunción final, en tanto que las evaluaciones emitidas por clase $y$, especialmente, el docente, reflejan discrepancias que llegan a superar el $10 \%$ de la escala de medición.

El desarrollo de la investigación ha puesto de relieve la importancia de realizar ajustes para garantizar la eficacia de la propuesta: es necesario realizar un mayor trabajo previo con el alumnado para que comprenda el alcance y significado de la tarea, ya que evaluar es para el profesorado en formación una cuestión especialmente ingrata, a la que intentan resistirse desde planteamientos todavía discentes; es crucial discernir para cada planteamiento si los grupos deben ser dirigidos o no, y bajo qué criterios en su caso, ya que la experiencia ha reflejado una clara tendencia a la homogeneidad por especialidades, lo que puede constituir un elemento relevante desde la perspectiva de juicio de mayorías/minorías y de las estrategias de comunicación, en tanto que el grueso estaba conformado por personas tituladas en Historia o Historia del Arte, y de forma marginal por Filosofía y Geografía.

Finalmente, consideramos que la investigación permite afirmar que la aplicación combinada de rúbricas y evaluación de $360^{\circ}$ supone una mejora en el proceso de evaluación en formación del profesorado, al incrementar el número de variables significativas y las relaciones entre ellas, permitiendo detectar patrones y relaciones internas de alto interés, difícilmente detectables de otro modo. Esto hace posible, además, un análisis más profundo de aspectos no fácilmente controlables por el docente, como la dinámica interna de grupos y el trabajo no supervisado, así como ayuda a fortalecer el compromiso de los futuros docentes con una fase clave del proceso educativo como es la evaluación, desde la experiencia práctica. 


\section{Referências bibliográficas}

Aguilar-Cuesta, Á I., \& Martínez-Romera, D. D. (2017). Utilidad de la metodología científica en la investigación educativa del profesorado en formación: Reflexión a partir de un estudio de caso en ciencias sociales sobre atención a la diversidad. CPU-E, Revista De Investigación Educativa, 1(1), 54-74. Retrieved from https://goo.gl/PH5TvZ

Agrawal, R., \& Srikant, R. (1994). Fast algorithms for mining association rules in large databases. Proceedings of the 20th International Conference on very Large Data Bases, 478-499. San Francisco (EEUU): Morgan Kaufmann Publishers Inc

Alsina, Á. y Batllori, R. (2015). Hacia una formación del profesorado basada en la integración entre la práctica y la teoría: una experiencia en el Prácticum desde el modelo realista. Revista de Investigación en la Escuela, 85, 5-18.

Arribas-Estebaranz, J. M., Manrique-Arribas, J. C., \& Taberner-Sánchez, B. (2016). Instrumentos de evaluación utilizados en la formación inicial del profesorado y su coherencia para el desarrollo de competencias profesionales en los estudiantes: visión del alumnado, egresados y profesorado. Revista Complutense de Educación, 27(1), 237. https://doi:10.5209/rev_RCED.2016.v27.n1.45724

Bisquerra-Alzina, R., Martínez-Olmo, F., Obiols-Soler, M., \& Pérez-Escoda, N. (2006). Evaluación de $360^{\circ}$ : una aplicación a la educación emocional. Revista de Investigación Educativa, vol. 24, no 1. Retrieved from https://goo.gl/NL5Gzd

Brutus, S., \& Gorriti, M. (2005). La Evaluación Multifuente Feedback 360. Revista de Psicología del Trabajo y de las Organizaciones, 21(3), 235-252. Retrieved from https:// goo.gl/KeIIwG

Cabero, J., \& Barroso, J. (2016). ICT teacher training: A view of the TPACK model. Revista Cultura Y Educación, 28(3), 633-663. https://doi:10.1080/11356405.2016.1203 526

Cachón-Zalagaz, J., López-Manrique, I., Romero-Granados, S., Zagalaz-Sánchez, M.L., y González-González de Mesa, C. (2015). Opinión de docentes y estudiantes del máster de secundaria sobre las aportaciones de este a la formación del profesorado, la calidad docente y los intereses personales. Magister: Revista miscelánea de investigación, 27(1), p. 1-10 (2015). http://dx.doi.org/10.1016/j.magis.2015.03.001

Cano, E. (2015). Las rúbricas como instrumento de evaluación de competencias en educación superior: ¿uso o abuso? Profesorado, Revista De Curriculum Y Formación Del Profesorado, 19(2), 265-280. Retrieved from https://goo.gl/KeIIwG

Cebrián-de-la-Serna, Manuel, Cebrián-Robles, D., \& Serrano-Puerto, J. (2015). Metodología para evaluar el impacto de las erúbricas y las anotaciones de vídeo en las prácticas externas. En Investigar con y para la sociedad (pp. 1457-1464). ISBN 97884-686-6906-9. España: AIDIPE. 
La evaluación de diseños de intervención para el Prácticum como instrumento de formación e investigación en el Máster de Profesorado de Ciencias Sociales.

Cebrián-de-la-Serna, Manuel, \& Bergman, M. E. (2014). Evaluación formativa con e-rúbrica: aproximación al estado del arte. REDU : Revista de Docencia Universitaria, 12(1), 15-22. Retrieved from https://goo.gl/bZfdmf

Cebrián-de-la-Serna, Manuel. (2014). eRubrics in cooperative assessment of learning at university/las eRúbricas en la evaluación cooperativa del aprendizaje en la universidad. Revista Comunicar (English Edition), 22(43), 153-160. Retrieved from https://goo.gl/XoEDZU

Cohen, L., Manion, L., \& Morrison, K. (2011). Research methods in education (7. ed., rewritten, expanded and updated ed.). London [u.a.]: Routledge.

Curiel-Marín, E., \& Fernández-Cano, A. (2015). Análisis Cienciométrico de Tesis Doctorales Españolas en Didáctica de las Ciencias Sociales (1976-2012). Revista Española de Documentación Científica, 38(4), e110. Retrieved from https://goo.gl/nCdtw6

Egbert, J., \& Sanden, S. (2014). Foundations of education research (1. publ. ed.). New York [u.a.]: Routledge.

Esteve-Zarazaga, J. M. (2009). La formación de profesores: bases teóricas para el desarrollo de programas de formación inicial. Revista de educación, (350), 15-30. Retrieved from https://goo.gl/nuK1aO

Fuentes-Moreno, C. (2016). La formación inicial de los docentes de Historia en Educación Secundaria. Revisión del Máster de Formación del Profesorado. Revista Andamio, v. 2, no 4, p. 35-49.

González-Ballesteros, M. y Fernández-Lozano, P. (2014). Procedimientos de evaluación de los formadores de profesores de primaria y de secundaria. Un estudio cualitativo. INIFAD Revista de Psicología, no 1, vol. 7, pp. 171 - 178. http://dx.doi.org/10.17060/ijodaep.2014.n1.v7.788

González-Faraco, J. C., Pérez-Moreno, H. M., \& Jiménez-Vicioso, J. R. (2011). El nuevo modelo formativo del profesorado de Educación Secundaria y su proceso de implantación en las Universidades Andaluzas. Fuentes: Revista de la Facultad de Ciencias de la Educación, 11, 67-85. Retrieved from https://goo.gl/GNbIWU

Laufer, B., \& Ravenhorst-Kalovski, G. C. (2010). Lexical threshold revisited: Lexical text coverage, learners' vocabulary size and reading comprehension. Reading in a Foreign Language, 22(1), 15. Retrieved from https://goo.gl/YNbDIz

Liu, B., Hsu, W., \& Ma, Y. (1998). Integrating classification and association rule mining. Proceedings of the Fourth International Conference on Knowledge Discovery and Data Mining, 80-86. New York: Association for the Advancement of Artificial Intelligence. 
Lorenzo-Vicente, J. A., Muñoz-Galiano, I. M., \& Beas-Miranda, M. (2015). Modelos de formación inicial del profesorado de educación secundaria en españa desde una perspectiva europea. Revista Complutense De Educación, 26(3) https://doi:10.5209/rev_RCED.2015.v26.n3.44866

Martinez, A., Mauri, T., Colomina, R., Agirre, N., Clarà, M., Bilbatua, M., Onrubia, J. y Lopez de Arana, E. (2016). Prácticas de reflexión colaborativa. Análisis de casos reales, relación teoría -práctica, comunidades de práctica. CIDUI, 2016, núm. 3, p. 1-11.

Martínez-Romera, D. D., Cebrián-Robles, D., \& Cebrián-de-la-Serna, M. (2016). Assessment of teaching skills with e-rubrics in master of teacher training. JETT, 1(Extra), 120-141. Retrieved from https://goo.gl/xBFHgR

Morin, E. (2001). El método. La naturaleza de la naturaleza (1st ed.). Madrid: Cátedra.

Morin, E. (1999). Los siete saberes necesarios para la educación del futuro (1st ed.) UNESCO.

Perales-Montolío, M. J., Jornet-Meliá, J. M., \& González-Such, J. (2014). Tendencias en las políticas de formación y evaluación del profesorado en la educación superior en España. RIEE. Revista Iberoamericana de Evaluación Educativa, 53-64 Retrieved from https://goo.gl/qUxOKT

Pérez-Pueyo, Á, Hortigüela-Alcalá, D., \& Gutiérrez-García, C. (2016). Reflexión sobre la evaluación en la formación inicial del profesorado en españa. en búsqueda de la concordancia entre dos mundos. IEYA, Revista Infancia Educación Y Aprendizaje, 2(2), 39-75.

Pérez-Torregrosa, A.B., Romero-López, M.A., Ibáñez-Cubillas, P. \& Gallego-Arrufat, M.J. (2017). Grado de satisfacción, utilidad y validez de la evaluación con rúbricas electrónicas durante el prácticum. Revista Prácticum, 2(1), 60-79.

Quivy, R., \& Van-Campenhoudt, L. (2005). Manual de investigación en ciencias sociales. México: Limusa.

Salerni, A. (2016). Il tirocinio universitario come strumento orientativo/formativo. Revista Practicum, Vol. 1(1) 80-98. ISSN 2530-4550

Schmitt, N., \& Schmitt, D. (2014). A reassessment of frequency and vocabulary size in L2 vocabulary teaching. Language Teaching, 47(4), 484-503. https://doi:10.1017/S0261444812000018 
La evaluación de diseños de intervención para el Prácticum como instrumento de formación e investigación en el Máster de Profesorado de Ciencias Sociales.

Sepúlveda, M.P., Gallardo, M., Mayorga, M.J. \& Madrid, D. (2017). La evaluación del Prácticum: un proceso clave en la construcción y reconstrucción del pensamiento práctico. ENSAYOS, Revista de la Facultad de Educación de Albacete, 32(1). http://dx.doi.org/10.18239/ensayos.v32i1.1368

Serrano-Rodríguez, R., \& Pontes-Pedrajas, A. (2015). Nivel de desarrollo de las competencias y objetivos generales del máster formación del profesorado de enseñanza secundaria. Perfiles Educativos, 37(150) Retrieved from https://goo.gl/roZYOH

Sternberg, R. J. (1999). Thinking styles (1. paperback ed. ed.). Cambridge [u.a.]: Cambridge Univ. Press.

Tejada-Fernández, J. (2011). La evaluación de las competencias en contextos no formales: dispositivos e instrumentos de evaluación. Revista de Educación, (354), 341-342. Retrieved from https://goo.gl/kYlvx2

Valdés-Puentes, R., Bolivar-Botia, A., \& Moreno-Verdejo, A. (2015). Una valoración de la formación inicial de profesores en España: El Máster en Educación Secundaria. Educação em Revista, 31(3) Retrieved from https://goo.gl/I1vi12

Viñao, A. (2013). Modelos de formación inicial del profesorado de educación secundaria en españa : Siglos XIX-XXI. Revista Española De Educación Comparada, (22), 19-37. http://dx.doi.org/10.5944/reec.22.2013.9321

Wood, P., \& Smith, J. (2016). Educational research: Taking the plunge. La Vergne: Independent Thinking Press. 